\title{
Ultrahigh Recovery of Fracture Strength on Mismatched Fractured Amorphous Surfaces of Silicon Carbide
}

Junfeng Cui, ${ }^{\dagger}$ Zhenyu Zhang, ${ }^{*} \dagger$ Haiyue Jiang, ${ }^{\dagger}$ Dongdong Liu, ${ }^{\dagger}$ Li Zou,,$\star \star \#$ Xiaoguang Guo, ${ }^{\dagger}$ Yao Lu," Ivan P. Parkin ${ }^{\S}$ and Dongming Guo ${ }^{\dagger}$ ${ }^{\dagger}$ Key Laboratory for Precision and Non-Traditional Machining Technology of Ministry of Education, Dalian University of Technology, Dalian 116024, China.

*School of Naval Architecture, State Key Laboratory of Structural Analysis for Industrial Equipment, Dalian University of Technology, Dalian 116024, China.

\#Collaborative Innovation Center for Advanced Ship and Deep-Sea Exploration, Shanghai 200240, China.

'Department of Chemistry, School of Biological and Chemical Sciences, Queen Mary University of London, London E1 4NS, UK.

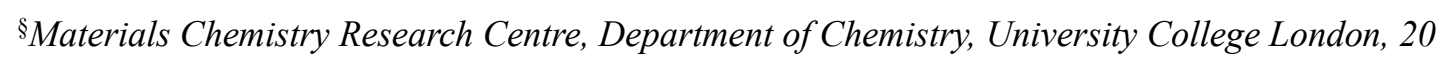
Gordon Street, London, WC1H 0AJ, UK.

ABSTRACT: Nanowires (NWs) have been envisioned as building blocks of nanotechnology and nanodevices. In this study, NWs were manipulated using a weasel hair, and fixed by conductive silver epoxy, eliminating the contaminations and damages induced by conventional beam depositions. The fracture strength of the amorphous silicon carbide was found to be $8.8 \mathrm{GPa}$, which was measured by in situ transmission electron microscopy nanomechanical testing, approaching the theoretical fracture limit. Here, we report that self-healing of mismatched fractured amorphous surfaces of brittle NWs was discovered. The fracture strength was found to be $5.6 \mathrm{GPa}$ on the mismatched fractured surfaces, recovering $63.6 \%$ of that of pristine NWs. This is an ultrahigh recovery, due to the limits of reconstruction of dangling bonds on the fractured amorphous surfaces and the mismatched areas. Simulation by molecular dynamics showed fracture strength recovery of $65.9 \%$ on the mismatched fractured amorphous surfaces, which is in good agreement with the experimental results. Healing on the mismatched fractured amorphous surfaces is by reorganization of Si-C bonds forming Si-C and Si-Si bonds. The potential energy increases $2.6 \mathrm{eV}$ in the reorganized Si-C bonds, and decreases by 3.2 and $1.9 \mathrm{eV}$, respectively, in the formed $\mathrm{Si}-\mathrm{C}$ and $\mathrm{Si}-$ 
Si bonds. These findings provide insights for the reliability, design and fabrication of high performance NW-based devices, to avoid catastrophic failure working in harsh and extreme environments.

Keywords: fracture strength; in situ TEM; nanomechanical test; molecular dynamics; $\mathrm{SiC}$

The yield strength of gold nanowires (NWs) is 100 times that of their corresponding bulk counterparts. ${ }^{1}$ Bending strength of silicon carbide (SiC) NWs is $53.4 \mathrm{GPa}$, which is substantially larger than that of nanotubes, approaching near the theoretical values. Therefore, defect-free $\mathrm{SiC}$ NWs appear to be the strongest material known. ${ }^{2}$ The maximum tensile strain of diamond NWs is up to $9 \%$, reaching the theoretical elastic limit. ${ }^{3} \mathrm{SiC}$ NWs show superplasticity with local elongation $>200 \% .{ }^{4}$ NWs exhibit exceptional mechanical properties, due to less defects and larger surface-to-volume ratio. ${ }^{3,5}$ These exceptional mechanical properties of NWs endow them, with real promise for use in nano-electromechanical systems (NEMS) with functionality. NWs have been expected as building blocks of nanotechnology, as well as next generation devices and materials. ${ }^{6,7}$ The mechanical properties of NWs are extremely significant for the reliability and optimum design and fabrication of NW-based devices. ${ }^{6}$ Nevertheless, nanomechanical testing of NWs is always challenging, because of difficulties to manipulate nanoscale materials..${ }^{67}$ As a result, the mechanical properties of NWs have been considerably overlooked, resulting in the underlying deformation mechanisms being elusive. ${ }^{8}$

It has been demonstrated that the band structure of semiconductors is altered by mechanical strain. Variation in the band structures strongly affects the electrical, optical and magnetic properties of semiconductors, and in turn is related to stain engineering. ${ }^{6,8}$ Silicon $(\mathrm{Si})$ and $\mathrm{SiC}$ are amongst the most important semiconductor materials. ${ }^{9} \mathrm{Si}$ works at temperatures less than $200{ }^{\circ} \mathrm{C}$, on account of the limits of its physical and chemical properties. ${ }^{10} \mathrm{SiC}$ has exceptional physical and mechanical properties, consisting of low density, high strength, high thermal conductivity, stability at high temperature, high resistance to shocks, low thermal expansion, wide (tunable) bandgap and chemical inertness. ${ }^{11,12}$ These properties make $\mathrm{SiC}$ an appropriate material serving at high temperature, high power, high frequency, radiation, and in harsh and extreme environments. ${ }^{4,13,14}$ $\mathrm{SiC}$ has become one of the most promising materials for power electronics, heterogeneous catalyst supports, hard- and biomaterials. ${ }^{11,12}$ In addition, mechanical properties of $\mathrm{SiC}$ are strengthened by 
stacking faults, i.e. defect engineering. ${ }^{8}$ Certain $\mathrm{SiC}$ defects have sharp optical and spin transitions, ${ }^{15}$ and therefore defect engineering is applied to explore and fabricate high performance $\mathrm{SiC}$ devices. ${ }^{15-}$ ${ }^{17}$ Amorphization of $\mathrm{SiC}$ takes place under laser shock, ${ }^{18}$ electron beam irradiation ${ }^{19}$ and deformation. ${ }^{4,9}$ Stacking faults serve as a precursor of amorphization. ${ }^{18}$ Crystalline-to-amorphous transition happens when SiC NWs are subjected to mechanical force, such as stain and defect engineering. ${ }^{4,9}$ Nonetheless, $\mathrm{SiC}$ is a brittle material, suffering from brittle fracture under larger stress. ${ }^{13}$ Hence, nanomechanical testing on amorphous NWs is significant to avoid catastrophic failure of brittle materials working in harsh and extreme environments. However, to the best of our knowledge, nanomechanical testing on mismatched fractured brittle NWs has not been reported. Additionally, focused-ion-beam (FIB) and electron beam depositions are widely employed to fix and connect NWs in transmission electron microscopy (TEM). ${ }^{20-22}$ Nevertheless, the two depositions readily induce damages and contaminations on the NWs that affect performance. ${ }^{22}$ Using deposition techniques on NWs, the measured results by nanomechanical testing have risks in reliability and robust design of high performance NW devices. It is necessary to develop an approach to fix and connect NWs to eliminate the contaminations and damages induced by conventional beam depositions.

Healing on fractured surfaces is important for the reliability and longevity of devices, and attracts much attention. ${ }^{23-30}$ Traditional self-healing in polymer materials consists of three elements: healing agent, a fiber to encapsulate the healing agent and a procedure for hardening the healing agent. ${ }^{23}$ High temperature, welding and oxidation are used to heal fractured ceramics. ${ }^{24,25}$ Nanoparticles and chemical fuel are employed to heal microscopic cracks of electrical wires. ${ }^{26}$ Compressive stress and electrochemical welding are applied to heal single elemental metal ${ }^{27}$ and semiconductor $^{28}$ NWs, respectively. Self-healing on polymers, ceramics, metals and semiconductors has not been reported. Partially fractured gallium arsenide (GaAs) NWs are restored though an amorphous layer without nanomechanical testing. ${ }^{31}$ Thus, self-healing on mismatched fractured brittle materials has not been demonstrated.

In this study, a setup was developed to fix and connect NWs using a weasel hair in air at room temperature, eliminating the damages and contaminations generated by traditional beam depositions. Self-healing on mismatched fractured brittle NWs is confirmed by nanomechanical testing. The 
healing mechanism on mismatched fractured amorphous semiconductors was elucidated by molecular dynamics (MD) simulations.

\section{RESULTS}
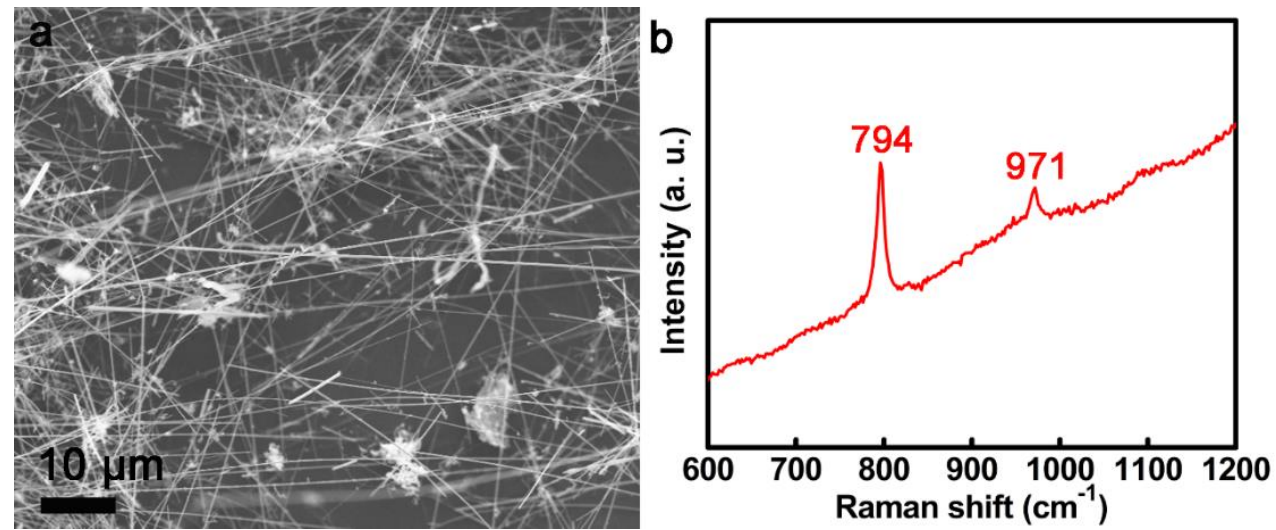

Figure 1 (a) SEM image and (b) its corresponding Raman spectrum of SiC NWs.
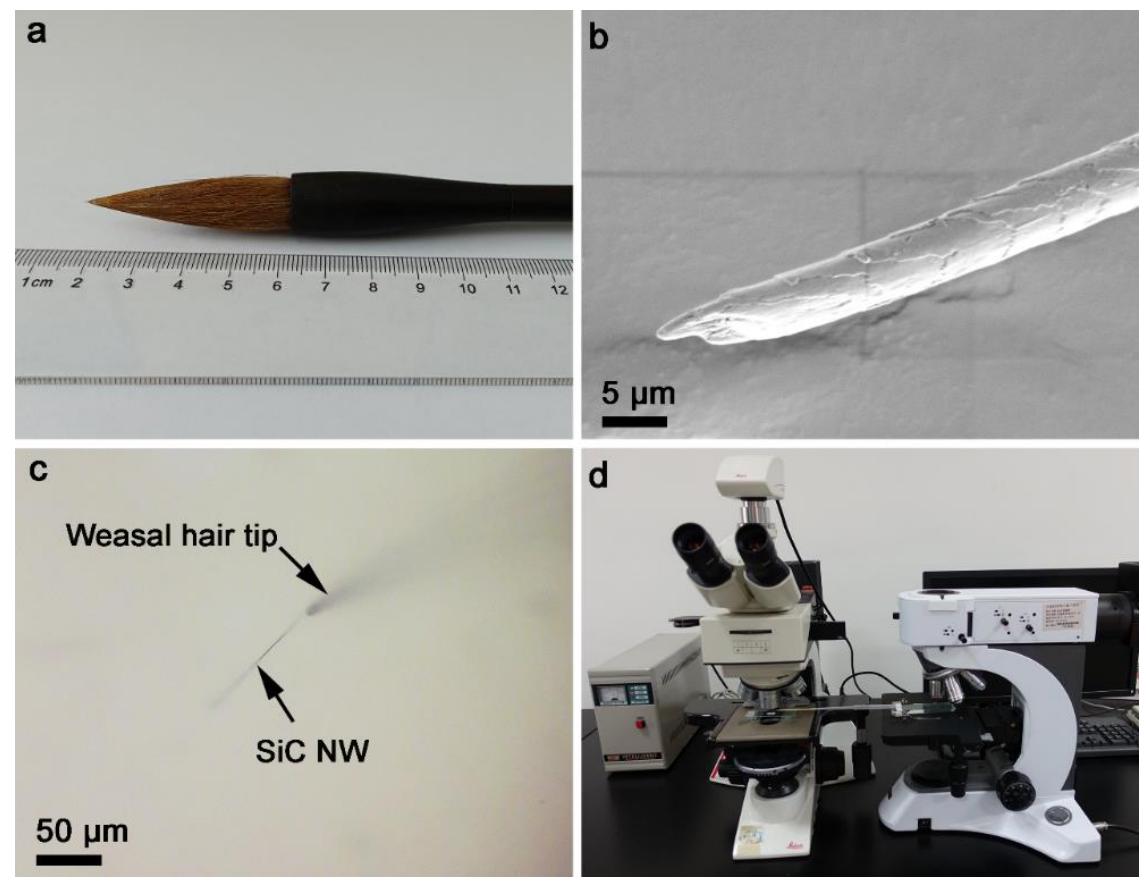

Figure 2 Photograph of (a) a weasel hair Chinese writing brush, (b) SEM image of its weasel hair tip, (c) optical image on picking up a NW using the tip, and photograph (d) of a developed setup.

Figure 1 illustrates the scanning electron microscopy (SEM) image and Raman spectrum of SiC NWs. The length of the NWs is hundreds of micrometers (Fig. 1(a)), exhibiting monocrystalline characteristics of 3C-SiC (Fig. 1(b)). ${ }^{32} \mathrm{~A}$ weasel hair tip was taken from a weasel hair Chinese writing brush (Fig. 2(a)). The radius of the weasel hair tip is hundreds of nanometers (Fig. 2(b)). The weasel hair tip has a tapered shape with diameters of several micrometers. It is used to 
manipulate a NW in a developed setup (Figs. 2(c) and 2(d)). An approach is proposed using a weasel hair to move and transfer a NW in the developed setup (Fig. 3), eliminating the contaminations and damage formed by conventional beam depositions. A weasel hair was employed to pick up a NW (Fig. 3(a)), and then put it on a push-to-pull (PTP) device (Fig. 3(b)). The NW is fixed on the PTP device by a conductive silver (Ag) epoxy (Fig. 3(c)). Nanomechanical tests were performed on the NW using a PTP device (Fig. 3(d)).
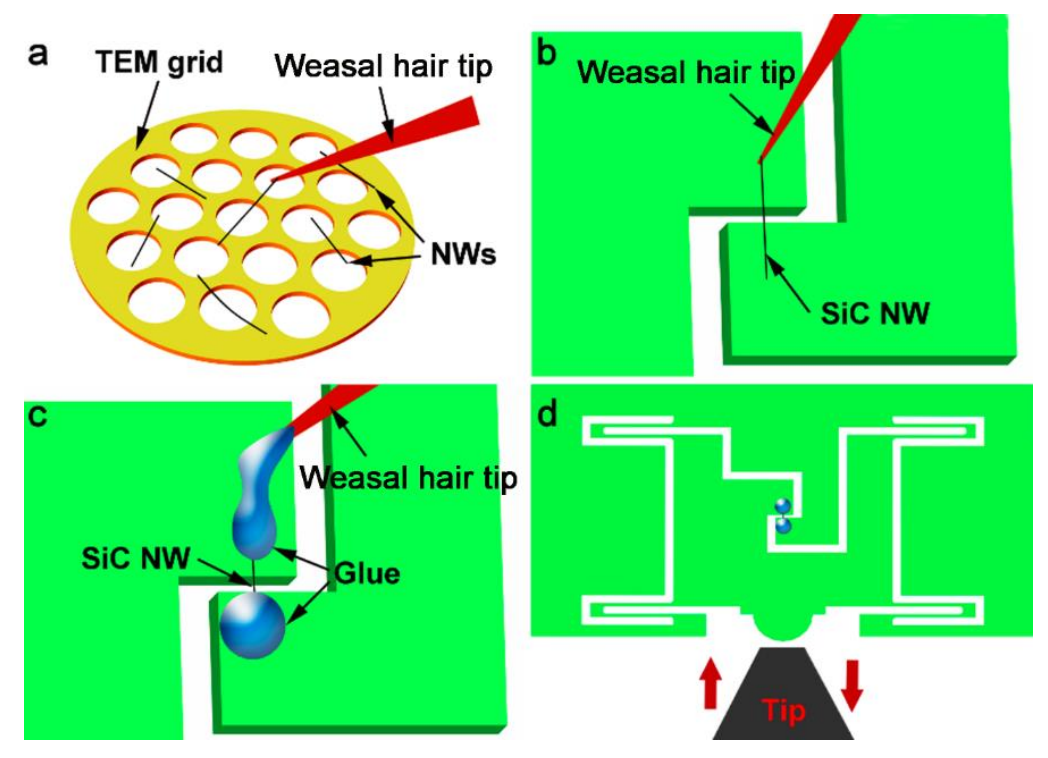

Figure 3 Schematic diagrams using (a) a weasel hair tip to (b) transfer a NW (c) fixed by conductive Ag epoxy on (d) a PTP device.
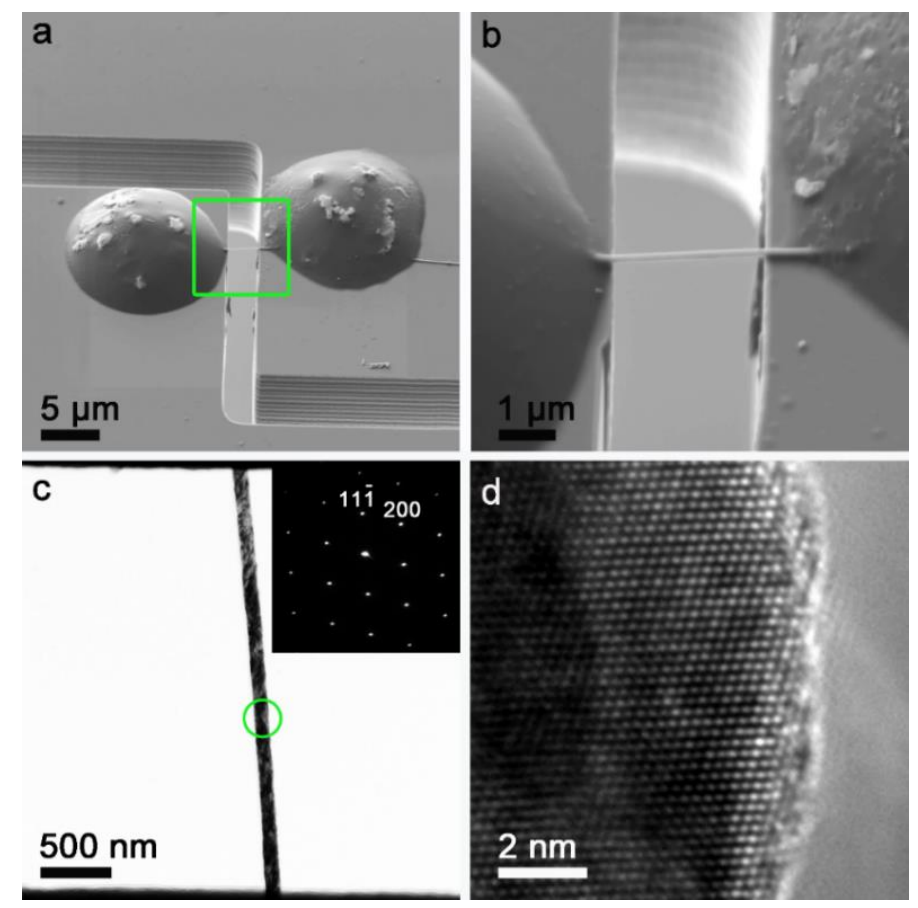

Figure 4 SEM (a), (b) and TEM (c), (d) images of a single crystal SiC NW at (a), (c) low and (b), 
(d) high magnifications fixed by conductive Ag epoxy on a PTP device. (b) is the magnified area taken from a green square in (a). Inset in (c) shows its corresponding SAED pattern.

Figure 4 shows the SEM and TEM images of a SiC NW fixed by conductive Ag epoxy on a PTP device. A SiC NW is fixed well on the PTP device, as depicted in Figs. 4(a) and 4(b). It looks very clean without contaminations formed by conventional beam depositions (Fig. 4(b)). The SiC NW has a diameter of about $100 \mathrm{~nm}$ (Fig. 4(c)), and the inset reveals monocrystalline characteristics by selected area electron diffraction (SAED) pattern. This is consistent with the single crystal lattice by the high resolution TEM (HRTEM) image in Fig. 4(d). An amorphous layer is approximately 0.5 nm outside the NW in Fig. 4(d) due to oxidization in air, which is in good agreement with the clean surface in Fig. 4(b).

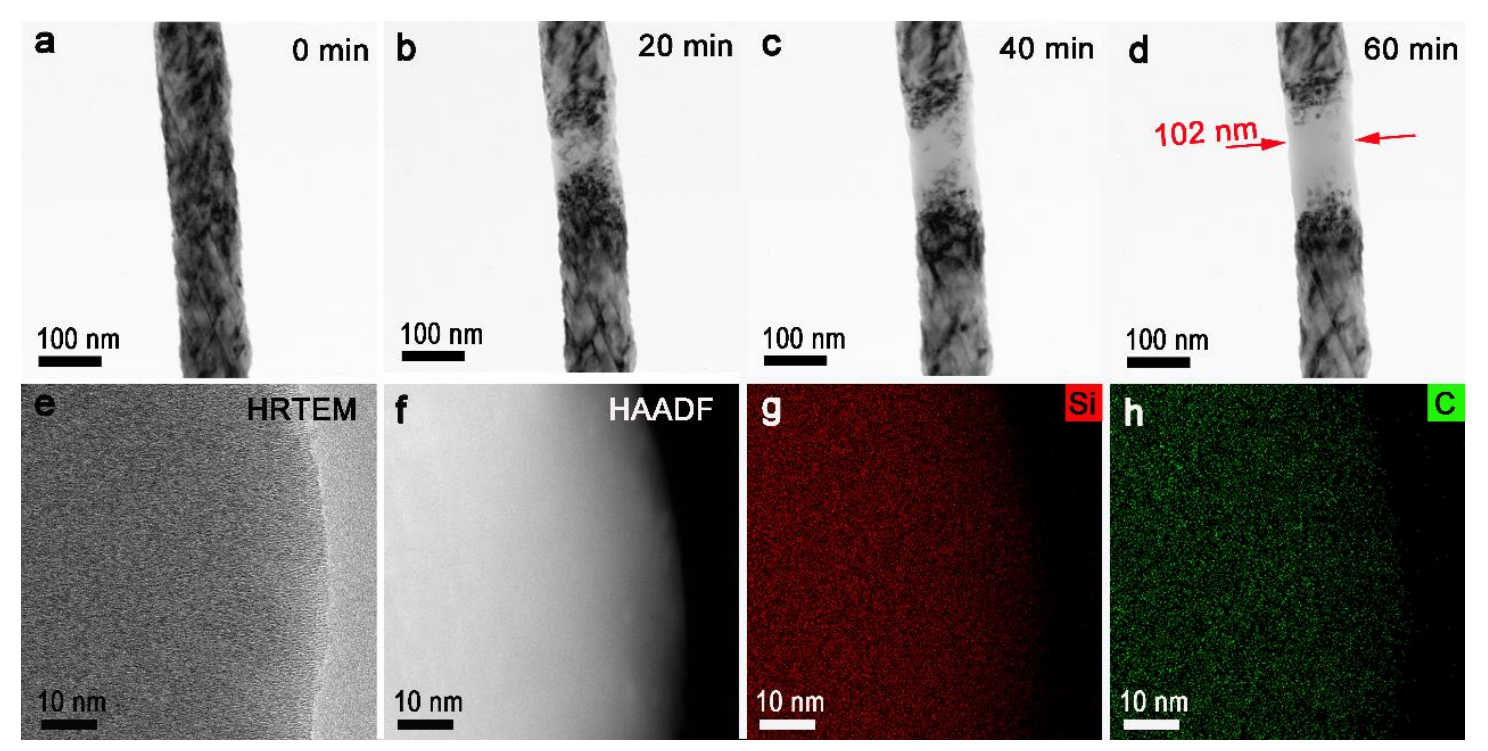

Figure 5 TEM images of a single crystal SiC NW irradiated by electron beam for (a) 0 , (b) 20, (c) 40, and (d) 60 minutes at a current density of $7.38 \mathrm{~A} / \mathrm{cm}^{2}$, (e) HRTEM, (f) HAADF-STEM images, and EDS tomography of (g) Si and (f) C elements around the area marked by the right arrow in (d).

Transformation from a single crystal to amorphous phase is illustrated in Fig. 5. Amorphous transformation accompanies a change of color from dark grey to white. Under the irradiation of the electron beam, partial amorphous transformation occurs after 20 minutes (Fig. 5(b)). With increasing irradiation time to 40 minutes, the amorphous phase becomes larger (Fig. 5(c)). When the irradiation time is 60 minutes, transformation from crystalline to amorphous phase is complete and the length is about $100 \mathrm{~nm}$ (Fig. 5(d)). The fractured amorphous phase is $102 \mathrm{~nm}$ in diameter for the in situ TEM fracture tests, as marked by a pair of red arrows in Fig. 5(d). HRTEM image 
illustrates the amorphous characteristics in Fig. 5(e). High angle annular dark field (HAADF) scanning TEM (STEM) image reveals the uniform density of amorphous SiC in Fig. 5(f). EDS tomography performed the compositional mapping of Si and $\mathrm{C}$ elements, to identify the elemental distribution in amorphous $\mathrm{SiC}$. $\mathrm{Si}$ and $\mathrm{C}$ elements distribute uniformly in amorphous $\mathrm{SiC}$, as depicted in Figs. 5(g) and 5(h), respectively.

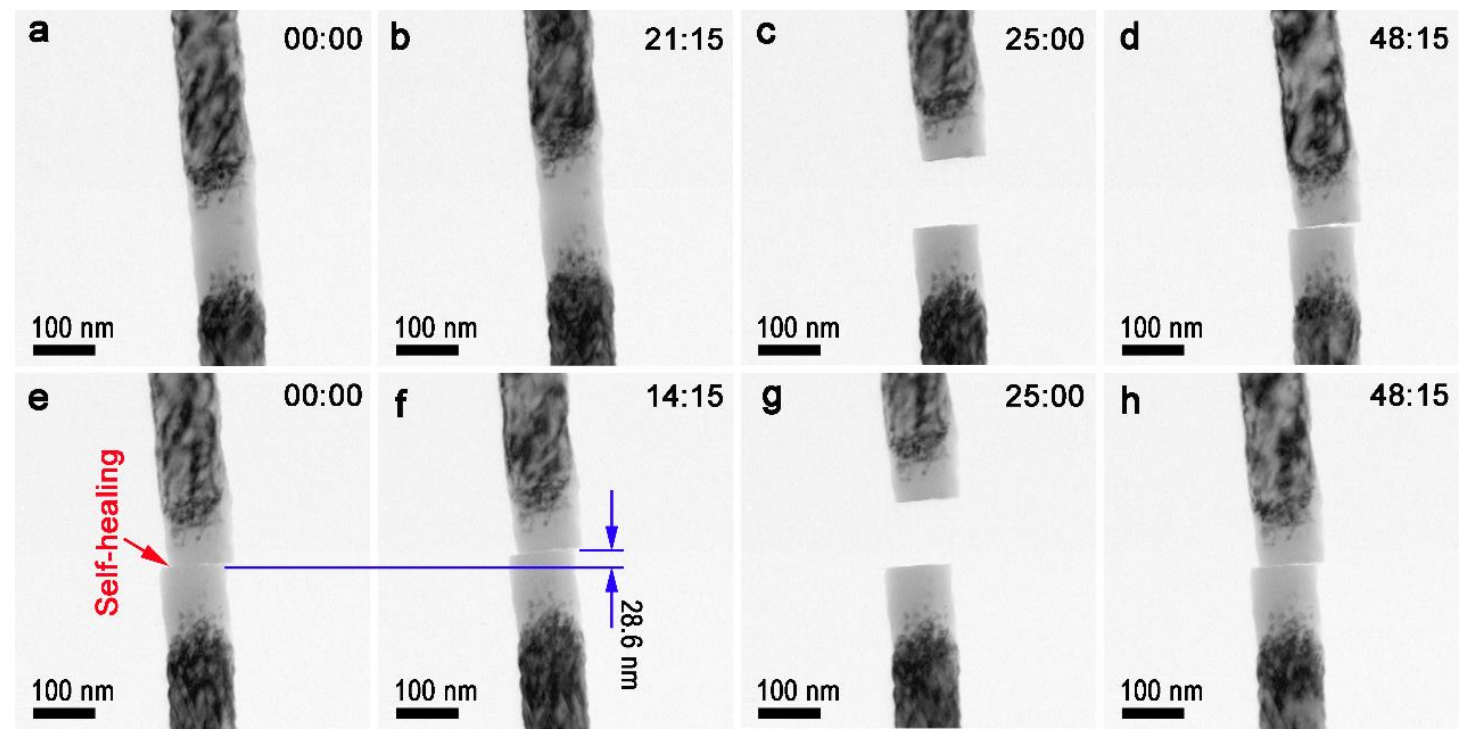

Figure 6 Snapshots of TEM images for the first (a), (b), (c), (d) and second (e), (f), (g), (h) fracture in an amorphous SiC NW prior to (d) and after (e) self-healing, before (b), (f) and after (c), (g) fracture and prior to (a), (e) and after (d), (h) loading.

Figure 6 pictures the snapshots of TEM images for the first and second fracture in an amorphous SiC NW. After fracture, two fractured amorphous surfaces are completely separated, as found in Fig. 6(c). Amorphous phase prefers to exhibit plastic characteristic under deformation, resulting in the compressive stress on the mismatched fractured surface. ${ }^{33}$ To exclude the effect of compressive stress, the two fractured amorphous surfaces were placed in contact after unloading with the force of zero (Fig. 6(d)), as measured and confirmed by the in situ TEM nanomechanical test system. The duration of healing is around 20 minutes as measured by TEM at room temperature, in which the electron beam was shut off. This removes the effect of electron beam on the healing, i.e. self-healing. After healing, the gap between two mismatched fractured surfaces diminishes drastically (Fig. 6(e)), compared to that before healing. Prior to fracture after self-healing (Fig. 6(f)), the healed surfaces elongate $28.6 \mathrm{~nm}$ (Fig. 6(f)), indicating the ability to bear tensile force. In situ TEM dynamic nanomechanical tests are displayed in Movies S1 and S2 for the first and second 
fracture, respectively. It is demonstrated that self-healing takes place on the mismatched fractured surfaces of brittle NWs, which is significant for the reliability and robust design of NW-based devices to avoid catastrophic failure working under extreme environments.
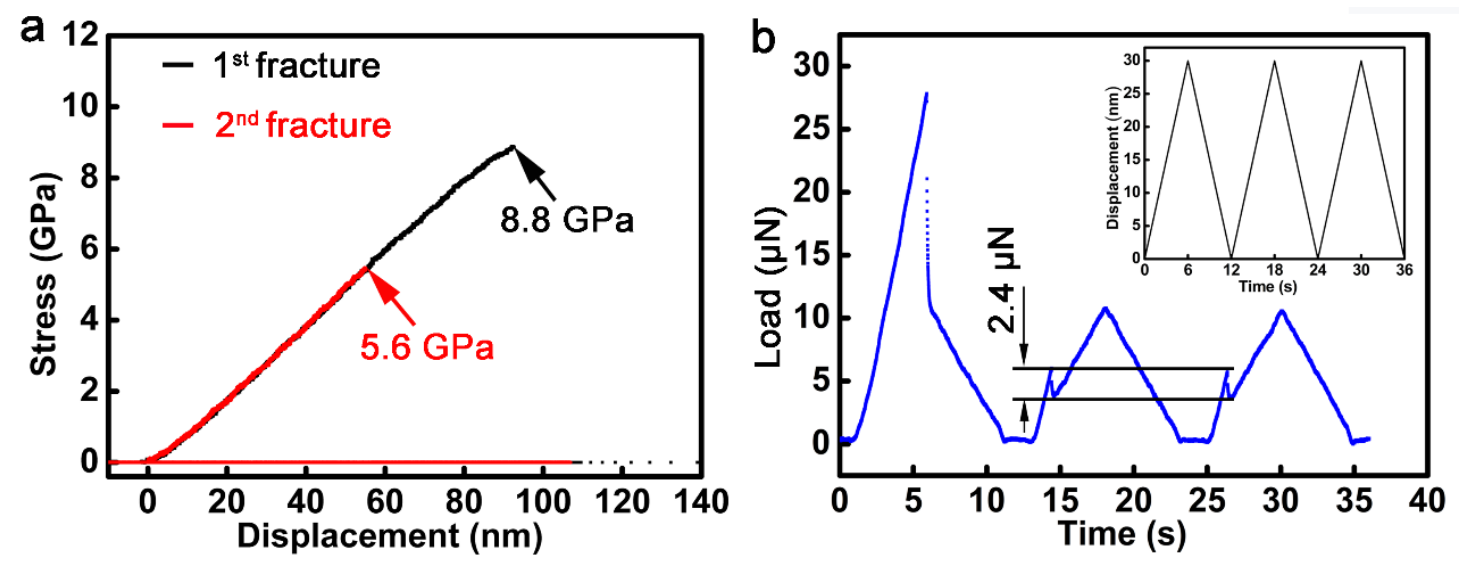

Figure 7 (a) Stress as a function of displacement for the in situ TEM fracture tests in Fig. 6, and (b) load as a function of time in fracture tests for a healing time of $2 \mathrm{~s}$. Inset in (b) shows the corresponding displacement as a function of time.

Figure 7(a) draws the stress as a function of displacement for the in situ TEM fracture tests in Fig. 6. Fracture strength is 8.8 and 5.6 GPa for the first and second fracture, respectively. Recovery of the fracture strength was measured at $63.6 \%$, which is an ultrahigh recovery on mismatched fractured surfaces of brittle NWs. To measure the ability of healing on mismatched fractured surfaces, a continuous displacement-controlled mode is shown in the inset of Fig. 7(b). In situ TEM dynamic fracture tests for a healing time of $2 \mathrm{~s}$ is displayed in Movie S3. The fracture force keeps stable at $2.4 \mu \mathrm{N}$ after healing for $2 \mathrm{~s}$, as illustrated in Fig. 7(b).

Figure 8 depicts the HRTEM images prior to and after self-healing on the mismatched fractures surfaces. Prior to self-healing, a crack is observed as shown in Fig. 8(a). After self-healing, the crack disappears, and the two fractured surfaces are healed well (Fig. 8(b)). Fast Fourier transformation (FFT) patterns show the amorphous characteristics in the insets of Fig. 8, indicating the absence of crystallites prior to and after self-healing. 

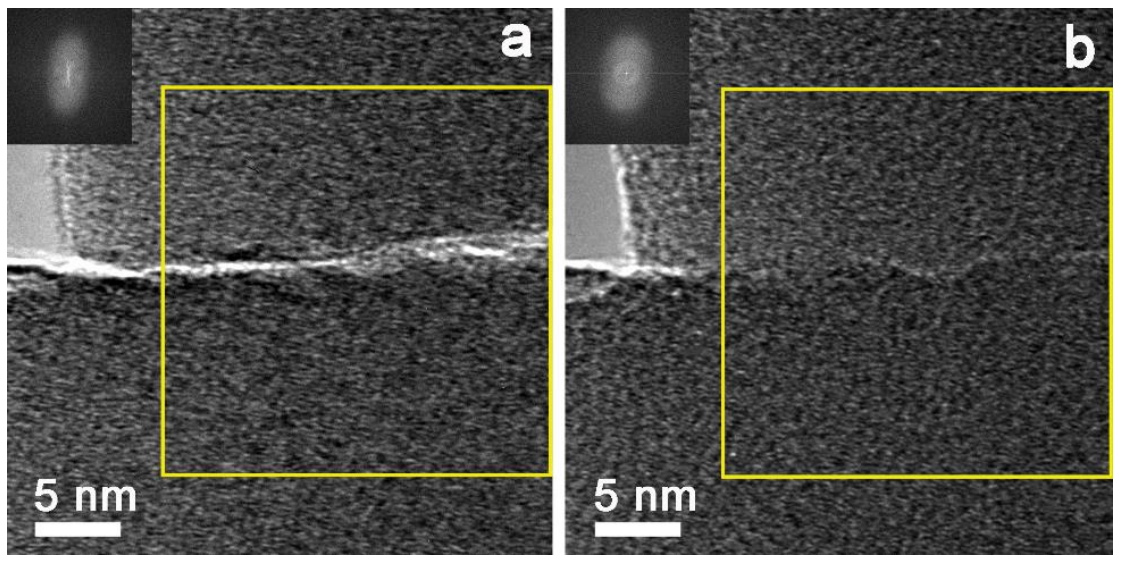

Figure 8 HRTEM images prior to (a) and after (b) self-healing magnified areas of left side on the mismatched fractured surfaces in Fig. 6(d) and Fig. 6(e), respectively. Insets show the FFT patterns taken from the corresponding areas marked by yellow squares.

\section{DISCUSSION}

In Fig. 1(b), Raman scattering signals at 794 and $971 \mathrm{~cm}^{-1}$ derive from transverse and longitudinal optical phonon modes, respectively, revealing obvious single crystal characteristics of 3C-SiC. ${ }^{32}$ After irradiation by an electron beam for $1 \mathrm{~h}$, an amorphous transformation happens as shown in Fig. 5(d). A NW was moved and transferred by a weasel hair, and then fixed by conductive Ag epoxy in air (Figs. 2 and 3). This approach eliminates contaminations induced by traditional beam depositions. ${ }^{20-22}$ Healed mismatched amorphous fractured surfaces of a NW are observed in Fig. 8(b). Healing on mismatched amorphous fractured brittle NWs was characterized and measured, by in situ TEM fracture tests, as illustrated in Figs. 6, 7 and 8. This is different from blurry TEM images of partially fractured NWs. ${ }^{31}$ TEM images and nanomechanical tests are direct experimental evidence for self-healing on mismatched fractured brittle NWs, which has not been reported previously.

There is a relationship between strength and elastic modulus of SiC. ${ }^{2}$ Fracture strength and elastic modulus of single crystal $\mathrm{SiC}$ are 23.8 and $448 \mathrm{GPa}$, respectively. ${ }^{34}$ As a consequence, fracture strength is $5.3 \%$ of the elastic modulus of $\mathrm{SiC}$. Elastic modulus of amorphous $\mathrm{SiC}$ is 170 $\mathrm{GPa},{ }^{35}$ and therefore theoretically predicted fracture strength is $9 \mathrm{GPa}$ for amorphous SiC. Fracture strength, $\sigma_{\mathrm{F}}$ is calculated, ${ }^{2}$

$\sigma_{\mathrm{F}}=\mathrm{F}_{\max } / \mathrm{A}$ 
where $\mathrm{F}_{\max }$ is the maximum force at fracture, and $\mathrm{A}$ is the area of fractured surface. Force and displacement are calibrated before calculating fracture strength through subtracting those applied on the PTP device. The measured fracture strength is $8.8 \mathrm{GPa}$ for amorphous SiC NWs, approaching the theoretical limit of fracture strength. This is attributed to that the amorphous $\mathrm{SiC}$ was prepared in vacuum of TEM, and then measured by in situ TEM nanomechanical system. From the preparation to measurement, the amorphous $\mathrm{SiC}$ is always in vacuum, absent contaminations and oxidation generated by traditional exposure in air. Furthermore, the amorphous $\mathrm{SiC}$ was produced from single crystal NWs with less defects and larger surface-to-volume ratio, ${ }^{3,5}$ which contributes for the high facture strength of amorphous SiC. After self-healing, fracture strength on mismatched fractured amorphous NWs is $5.6 \mathrm{GPa}$, restoring $63.6 \%$ that of pristine counterparts. This is an ultrahigh recovery of fracture strength on mismatched fractured brittle NWs, which provides insights to improve the stability, design and fabricate high performance NW-based devices. Moreover, it is also beneficial to avoid catastrophic failure of NW devices working in extreme environments.

NWs have a large surface-to-volume ratio, compared with bulk counterparts. This induces dangling bonds after fracture are much more prevalent on the fractured amorphous NWs. SiC is a polar semiconductor, and there are opposite charges on the fractured surfaces. ${ }^{36}$ Electrostatic force takes place on the fractured surfaces, facilitating rebonding of mismatched NWs ${ }^{31}$ Amorphous SiC has a disordered phase, which contributes to self-healing on mismatched fractured amorphous SiC. Disordered dangling bonds could bond directly, without the need to reorient as that required in crystals for self-healing. Atomic rearrangement benefits self-healing on fractured amorphous SiC. The driving force of atomic rearrangement is to reduce the system energy by reducing the surface area, as well as surface energy. ${ }^{31}$ Little has been reported for self-healing occurring on a NW with diameters more than $12 \mathrm{~nm} .{ }^{31,37}$ This might be attributed to the contaminations made by traditional beam depositions. The contaminations change the mechanical and electronic properties of NWs. ${ }^{38}$ In this work, NWs were manipulated and fixed by a weasel hair and conductive Ag epoxy in a developed setup, respectively, eliminating the contaminations induced by traditional beam depositions. Dangling bonds play the most important role for the self-healing on the fractured surfaces. NWs have a large surface-to-volume ratio, ${ }^{3,5}$ contributing tremendously on the self-healing 
of fractured surfaces. Dangling bonds on the fractured surfaces will be passivated in air by oxygen atoms, and therefore fractured surfaces can not be self-healed in air. For amorphous transformation irradiated by electron beam, the diameter of NWs can not exceed $150 \mathrm{~nm}$, otherwise edges of NWs will be removed by electron beam and the center is not transformed amorphously completely, as illustrated in Fig. 9. In Fig. 8, the fractured surfaces of NWs are not smooth at nanoscale, while they can be self-healed due to that the variation of height on the fractured surfaces is approximately 2 $\mathrm{nm}$. When the diameter of NWs is more than $150 \mathrm{~nm}$, a gap between the two fractured surfaces will form gradually due to the bigger variation of height, which can not be self-healed by dangling bonds. Ionization and displacement damages take place during electron irradiation. ${ }^{39}$ It is reported that the ionization damage decreases with increasing the accelerate voltage of electrons. However, in this study, the irradiation-induced amorphization speed increases with increasing the accelerate voltage of electrons. As a result, the amorphization mechanism in present study is attributed to the accumulation of displacement damage, rather than the ionization effect. This is in good agreement with those previously reported irradiated by electrons. ${ }^{39}$

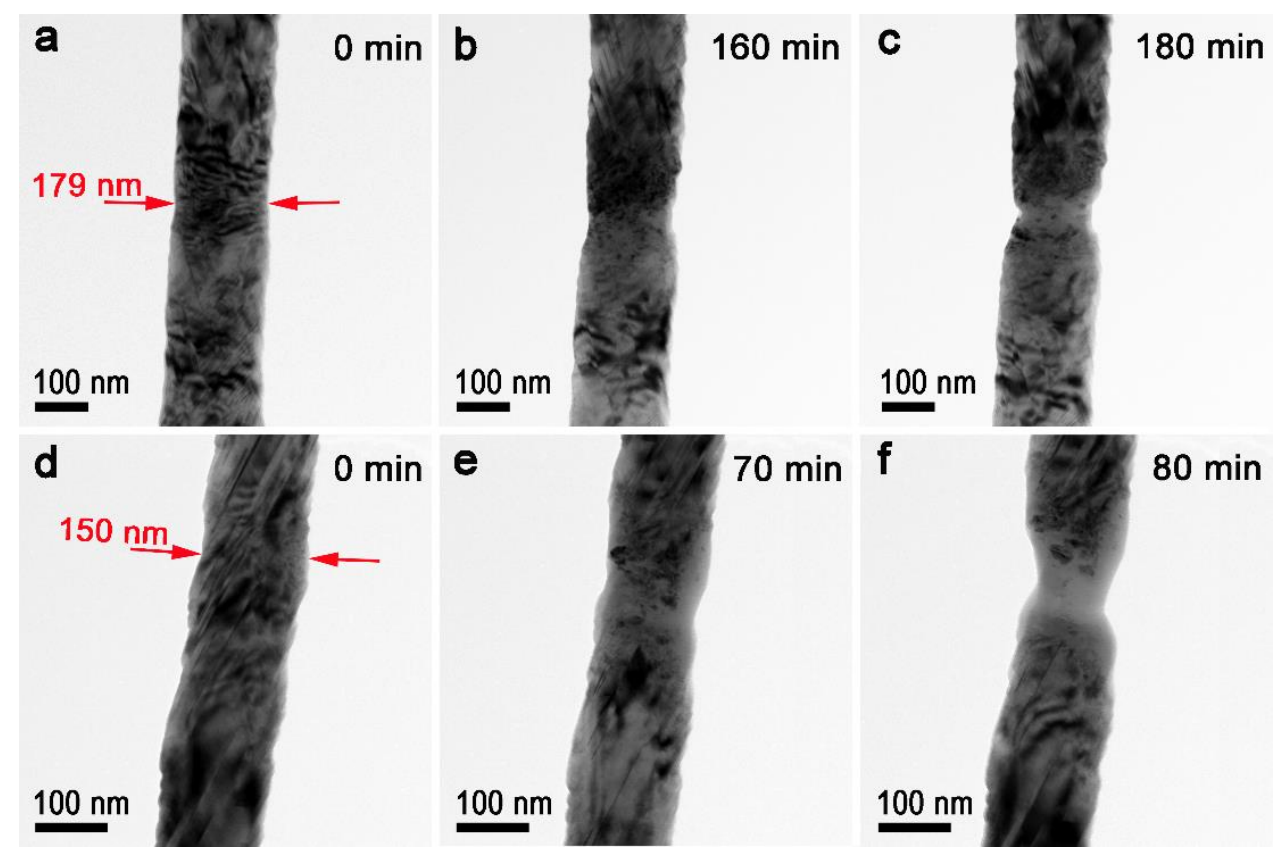

Figure 9 TEM images of single crystal SiC NWs with diameters of $179 \mathrm{~nm}$ irradiated by electron beam for (a) 0, (b) 160, (c) 180 minutes and $150 \mathrm{~nm}$ for (d) 0 , (e) 70, (f) 80 minutes at a current density of $7.38 \mathrm{~A} / \mathrm{cm}^{2}$. 

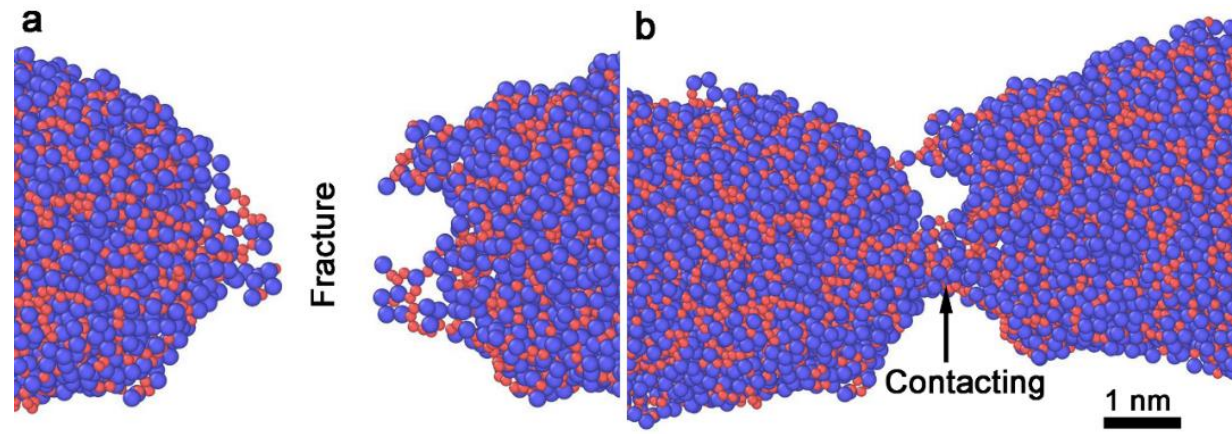

C

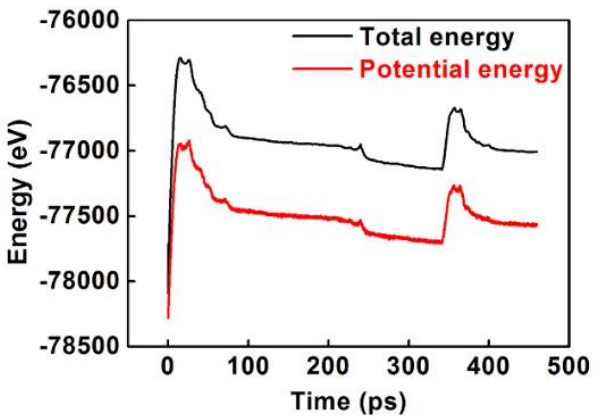

d

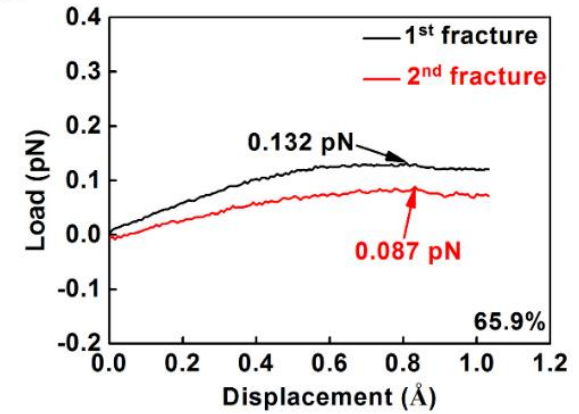

Figure 10 Snapshots (a) after the first fracture, (b) during contacting prior to the second fracture test, (c) energy as a function of time, and (d) load-displacement curves of the two fracture tests in MD simulations.

To elucidate the healing mechanism on mismatched fractured amorphous surfaces of brittle NWs, MD simulations are illustrated in Fig. 10. The NW is fractured completely (Fig. 10(a)), and mismatched amorphous surfaces are constructed in Fig. 10(b). This is consistent with the experimental results in Fig. 6. The potential energy agrees well with total energy during the two fracture tests, as depicted in Fig. 10(c). Recovery of fracture strength on the two mismatched fractured surfaces is $65.9 \%$ (Fig. 10(d)), which is in good agreement with the experimental results in Fig. 7(a). In Fig. 10(c), the highest peak in the left resulted from the increase of strain energy during the first tensile test. In the first fracture, strain energy is released, leading to the decrease of energy. The small peak in the middle is derived from the broken of Si-C and Si-Si bonds, resulting in the increase of energy prior to contacting with the mismatched fractured amorphous surfaces. During healing on the mismatched fractured surfaces, rebonding of dangling bonds induces a decrease in energy. The higher peak on the right is formed by an increase in the strain energy during the second tensile test, and then the release of the stain energy during the fracture. Because the fracture strength is recovered to $65.9 \%$, the intensity of the right peak induced by the second fracture 
test is lower than that of the first one.
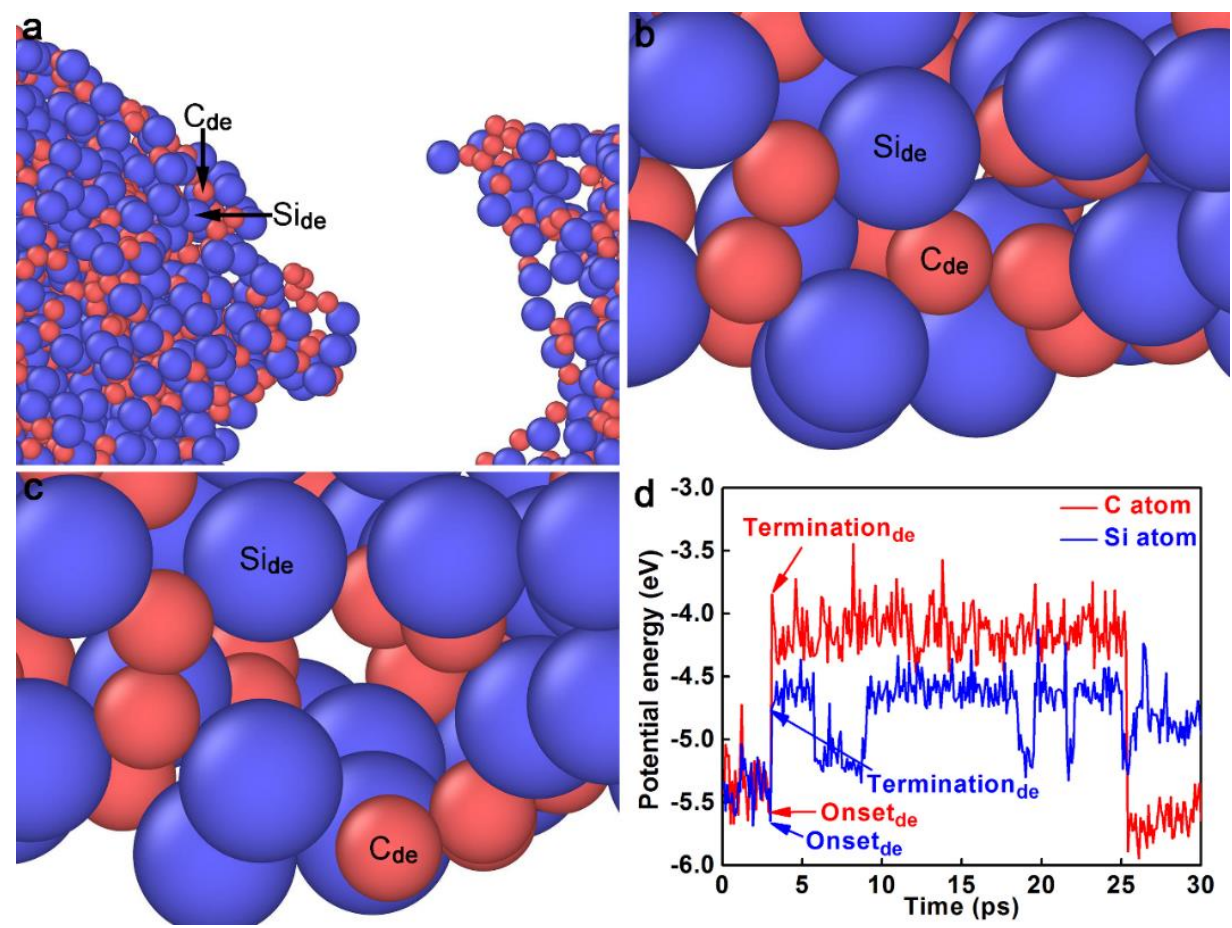

Figure 11 Snapshots of Si-C bonds (a), (b) prior to and after (c) broken, and (d) corresponding variation of potential energy for the two broken atoms before contacting on the mismatched fractured surfaces.

The breakage of the Si-C bonds takes place prior to contacting on the mismatched fractured amorphous surfaces, as depicted in Figs. 11(a), 11(b) and 11(c). During breakage, the potential energy increases for both the broken atoms, as illustrated in Fig. 11(d), which is consistent with the small peak in the middle of Fig. 10(c). The potential energy increases 1.7 and $0.9 \mathrm{eV}$ for the broken $\mathrm{C}$ and $\mathrm{Si}$ atoms, respectively, and a pair of $\mathrm{C}$ and $\mathrm{Si}$ atoms increase $2.6 \mathrm{eV}$ during the re-formation of Si-C bonds. 


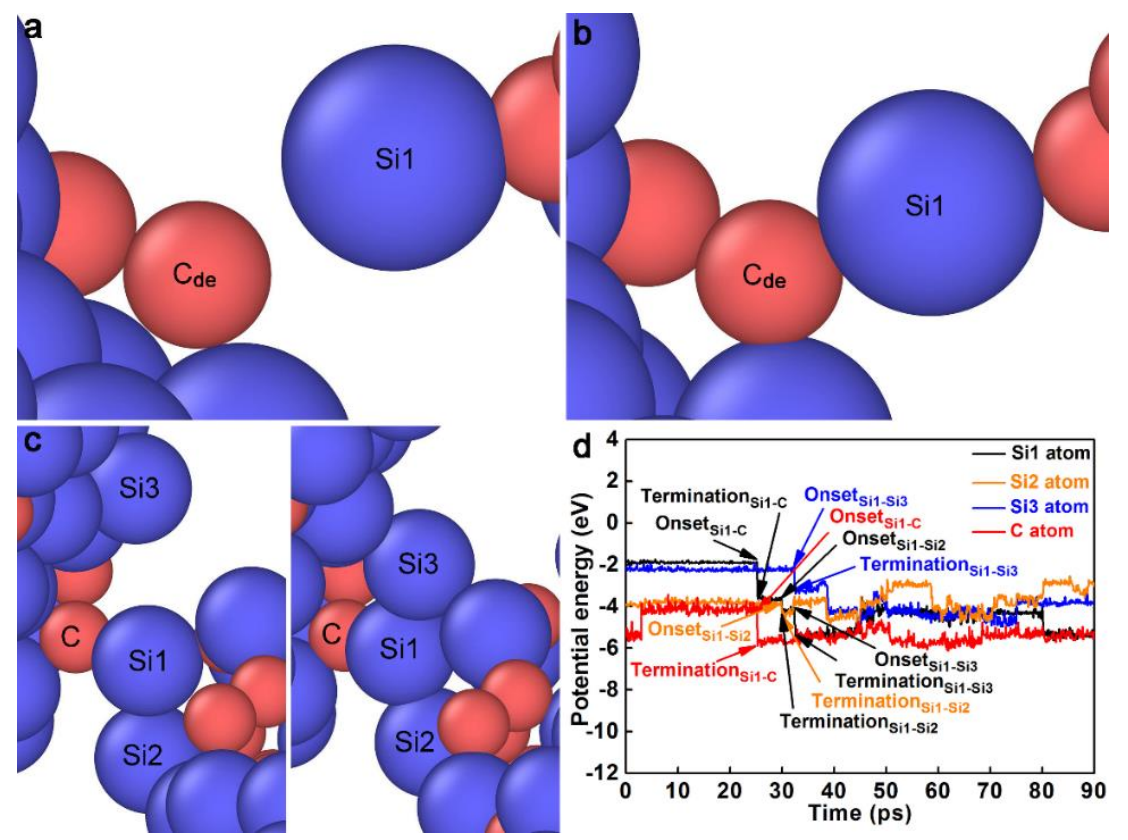

Figure 12 Snapshots prior to (a) and after (b) rebonding of Si-C bonds, (c) rebonding of Si-Si bonds, and their corresponding variations of potential energy during contacting on mismatched fractured surfaces.

Snapshots prior to and after re-forming of Si-C bonds are shown in Figs. 12(a) and 12(b), respectively. After rebonding with a $\mathrm{C}$ atom, $\mathrm{Si} 1$ atom rebonds with $\mathrm{Si} 2$ and $\mathrm{Si} 3$ atoms in a sequence as shown in Fig. 12(c). Figure 12(d) draws the variation of potential energy during rebonding between Si1 and C, Si2, Si3 atoms. Formation energy of C-Si and Si-Si covalent bonds consists of kinetic and potential energies. Potential energy of Sil atom decreases 1.6, 0.4, $1.4 \mathrm{eV}$, and the formation energy of Si-C and Si-Si covalent bonds decreases 2.8, 1.1 and $3.2 \mathrm{eV}$ during rebonding of Si1-C, Si1-Si2 and Si1-Si3 bonds, respectively. The $\mathrm{C}_{\mathrm{de}}$ atom in Figs. 12(a) and 12(b) is the same with both $\mathrm{C}_{\mathrm{de}}$ in Fig. 11 and $\mathrm{C}$ in Fig. 12(c), and its potential energy decreases $1.6 \mathrm{eV}$ during rebonding of Sil-C bonds. This is consistent with an increase of $1.7 \mathrm{eV}$ in potential energy in breaking Si-C bonds as shown in Fig. 11. The potential energy decreases 0.4 and $1.3 \mathrm{eV}$ for Si2 and $\mathrm{Si} 3$ atoms during bond formation with Si1 atom, respectively. Three pairs of atoms decrease potential energy by 3.2, 0.8 and $2.7 \mathrm{eV}$ during the formation of Si1-C, Si1-Si2 and Si1-Si3 bonds, respectively. The potential energy of the three pairs of covalent bonds decreases $6.7 \mathrm{eV}$, which is lower than their decrease of formation energy of $7.1 \mathrm{eV}$. On the fractured amorphous surfaces of $\mathrm{SiC}$, dangling bonds undergo reconstruction as illustrated in Figs. 11 and 12. Bond breakage 
increases the potential energy, resulting in a formation of dangling bonds. Bond formation decreases the potential energy, leading to the formation of Si-C and Si-Si bonds. Mismatching does not seem to affect the rebonding of the Si-C and Si-Si dangling bonds, decreasing the potential energy of NWs. It is, to our knowledge, an ultrahigh recovery of $63.6 \%$ of fracture strength on mismatched fractured surfaces, due to the limits of reconstruction of dangling bonds and mismatched areas of brittle NWs.

\section{CONCLUSIONS}

In summary, a setup was developed to move, transfer and fix NWs using a weasel hair and conductive Ag epoxy, to eliminate the contaminations and damages generated by traditional beam depositions. Self-healing is demonstrated on mismatched fractured amorphous surfaces of brittle NWs, as confirmed and measured by in situ TEM nanomechanical tests. Fracture strength recovers $63.6 \%$ on the mismatched fractured surfaces of NWs, which is an ultrahigh strength recovery and is related to the limits of mismatched areas and reconstruction of dangling bonds. MD simulations were performed to elucidate the healing mechanism on the mismatched fractured amorphous surfaces. The simulated results of MD are consistent with those seen experimentally. Breaking of $\mathrm{Si}-\mathrm{C}$ bonds happens prior to contacting on mismatched fractured amorphous surfaces. Si-C and $\mathrm{Si}$ Si bonds formation takes place during contact of the mismatched fractured surfaces. Bond breakage increases the potential energy, while bond formation decreases it. Healing is an effective way to decrease the energy of the NWs through rebonding of Si-C and Si-Si bonds. The outcomes shed light on robust design and fabrication of brittle NWs used in NEMS and nanodevices serving at high temperature, high power, high frequency and in harsh environments.

\section{EXPERIMENTS AND SIMULATIUONS}

Single crystal 3C-SiC NWs (Nanjing XFNANO Materials Tech Co., Ltd., China) were used for specimens of nanomechanical testing. SiC NWs were characterized by Raman spectra (Renishaw inVia Reflex, UK). A copper $(\mathrm{Cu})$ grid for TEM sample preparation was used with a diameter of $3 \mathrm{~mm}$, which was covered by a $\mathrm{C}$ film. A lighter was used to burn off the $\mathrm{C}$ film on the $\mathrm{Cu}$ grid to hold the NWs. A weasel hair was glued on a toothpick by conductive Ag epoxy (CircuitWorks ${ }^{\circledR}$ Conductive Epoxy, CW2400, Chemtronics ${ }^{\circledR}$, USA). The toothpick was inserted in a pipette dropper with one end glued by double faced adhesive tape on a glass block. The glass block 
was put on a mechanical stage of an optical microscope (NMM-800RF, Ningbo Yongxin Optics Co., Ltd., China). The mechanical stage was moved at millimeters and micrometers, which was controlled by coarse and fine focuses respectively. NWs were operated in another optical microscope (Leica DM2500 M, Germany). They were ultrasonically dispersed in alcohol solution for 3 minutes, and then dropped by a pipette on the $\mathrm{Cu}$ grid. After evaporation of the alcohol, the NWs were dispersed on the $\mathrm{Cu}$ grid. Weasel hair was inserted under a NW in a hole of the $\mathrm{Cu}$ grid, and then the NW was moved and transferred by the weasel hair from the $\mathrm{Cu}$ grid on a PTP device. Then, the NW was fixed by conductive Ag epoxy on the PTP device. Finally, the PTP device was mounted in an in situ TEM nanomechanical system (PI 95 TEM PicoIndenter, Bruker, USA) cooperated with a TEM (Talos F200X, ThermoFisher, Scientific, USA) operated at $200 \mathrm{kV}$. In situ TEM nanomechanical testing was performed at a displacement-controlled mode, and a loading speed was $5 \mathrm{~nm} / \mathrm{s}$.

A face-centered cubic (fcc) crystalline cylinder of $3 \mathrm{C}-\mathrm{SiC}$ was constructed with a diameter of $4 \mathrm{~nm}$ and a length of $12 \mathrm{~nm}$. The cylinder consisted of 14256 atoms. MD simulations were performed on the cylindrical model. The model was along [111], [-1-12] and [1-10] orientations for $\mathrm{X}, \mathrm{Y}$ and $\mathrm{Z}$ directions, respectively. Shrink-wrapped boundary conditions were applied to the $\mathrm{X}, \mathrm{Y}$ and Z directions. Canonical NVT ensembles including constant number, volume and temperature were used in the $\mathrm{MD}$ simulations. ${ }^{40}$ Blue and red colors represent Si and $\mathrm{C}$ atoms, respectively. Time step was 1 femtosecond (fs), and Tersoff potential was employed during MD calculations. The MD model was relaxed at $300 \mathrm{~K}$ for 20 picoseconds (ps). Firstly, the temperature was increased from 300 to $4000 \mathrm{~K}$ for $800 \mathrm{ps}$, and finally the temperature reached $4065.4 \mathrm{~K}$. Then, the temperature decreased dramatically for $10 \mathrm{ps}$, until reaching at $1160.5 \mathrm{~K}$. After a further decrease for $20 \mathrm{ps}$, the temperature was kept at a constant of $300 \mathrm{~K}$, and the MD model transformed from crystalline to an amorphous phase. ${ }^{41,42}$ Then, the amorphous model was conducted by fracture tests. During the test, the left side of $5 \mathrm{~nm}$ in length and right side of $4 \mathrm{~nm}$ in length were fixed, and the middle of $3 \mathrm{~nm}$ in length was used for the fracture tests simulation. During two fracture tests, the left side was kept stable invariably, and the right side was moved at a speed of $8 \mathrm{~m} / \mathrm{s}$. In the first fracture test, the right side was moved along the positive direction of $\mathrm{X}$ axis for $120 \mathrm{ps}$, until the amorphous model fractured completely. The fractured right side was moved along the positive direction of the $\mathrm{Y}$ axis 
for $10 \mathrm{ps}$, leading to the mismatched axes with a distance ranging from 6 to $8 \AA$ between the left and right fractured NWs. Then, the fractured right side was moved along the negative direction of $\mathrm{X}$ axis for $110 \mathrm{ps}$, contacting the fractured left side. After contacting, the two mismatched fractured NWs were relaxed for $100 \mathrm{ps}$. After relaxation, the second fracture test was started. The right side was moved along the positive direction of $\mathrm{X}$ axis for $120 \mathrm{ps,}$, and mismatched fractured amorphous NWs were completely fractured again.

\section{ASSOCIATED CONTENT}

\section{Supporting Information}

The Supporting Information is available free of charge on the ACS Publications website at DOI:

Details of videos on in situ TEM fracture tests for the first, second and third times (Movies S1-S3).

The authors declare no competing financial interest.

\section{AUTHOR INFORMATION}

\section{Corresponding Author}

*E-mail: zzy@dlut.edu.cn.

ORCID

Zhenyu Zhang: 0000-0002-2393-520X

\section{Author Contributions}

Z.Y.Z. and D.M.G. conceived the projects. J.F.C. and D.D.L. performed the experiments. Z.Y.Z. wrote the paper. H.Y.J. and X.G.G. conducted the MD simulations. Z.Y.Z., J.F.C., L.Z., Y.L. and I.P.P. analyzed the healing mechanism on mismatched fractured NWs. All authors discussed the results and commented on the manuscript.

\section{ACKNOWLEDGEMENTS}

Z.Y.Z. acknowledges the valuable discussions with Prof. Chun Tang from Jiangsu University on the MD simulations, and Y.L. is grateful for the financial support from the QMUL-SBCS start up. The authors acknowledge the financial supports from the National Key R\&D Program of China (YS2018YFA070119), Excellent Young Scientists Fund of NSFC (51422502), Science Fund for Creative Research Groups of NSFC (51621064), Changjiang Scholar Program of Chinese Ministry of Education, Program for Creative Talents in University of Liaoning Province (LR2016006), Distinguished Young Scholars for Science and Technology of Dalian City (2016RJ05), the Xinghai 
Science Funds for Distinguished Young Scholars and Thousand Youth Talents at Dalian University of Technology, Science Fund of State Key Laboratory of Tribology, Tsinghua University (SKLTKF17B19), Science Fund of State Key Laboratory of Metastable Materials Science and Technology, Yanshan University (201813), and the Collaborative Innovation Center of Major Machine Manufacturing in Liaoning.

\section{REFERENCES}

(1) Wu, B.; Heidelberg, A.; Boland, J. J. Mechanical Properties of Ultrahigh-Strength Gold Nanowires. Nat. Mater. 2005, 4, 525-529.

(2) Wong, E. W.; Sheehan, P. E.; Lieber, C. M. Nanobeam Mechanics: Elasticity, Strength, and Toughness of Nanorods and Nanotubes. Science 1997, 277, 1971-1975.

(3) Banerjee, A.; Bernoulli, D.; Zhang, H. T.; Yuen, M. F.; Liu, J. B.; Dong, J. C.; Ding, F.; Lu, J.; Dao, M.; Zhang, W. J.; Lu, Y.; Suresh, S. Ultralarge Elastic Deformation of Nanoscale Diamond. Science 2018, 360, 300-302.

(4) Zhang, Y. F.; Han, X. D.; Zheng, K.; Zhang, Z.; Zhang, X. N.; Fu, J. Y.; Ji, Y.; Hao, Y. J.; Guo, X. Y.; Wang, Z. L. Direct Observation of Super-Plasticity of Beta-SiC Nanowires at Low Temperature. Adv. Funct. Mater. 2007, 17, 3435-3440.

(5) Hu, Y. W.; Li, J.; Tian, J. F.; Xuan, Y.; Deng, B. W.; McNear, K. L.; Lim, D. G.; Chen, Y.; Yang, C.; Cheng, G. J. Parallel Nanoshaping of Brittle Semiconductor Nanowires for Strained Electronics. Nano Lett. 2016, 16, 7536-7544.

(6) Chen, Y. J.; An, X. H.; Liao, X. Z. Mechanical Behaviors of Nanowires. Appl. Phys. Rev. 2017, 4, 031104.

(7) Bernal, R. A.; Filleter, T.; Connell, J. G.; Sohn, K.; Huang, J. X.; Lauhon, L. J.; Espinosa, H. D. In Situ Electron Microscopy Four-Point Electromechanical Characterization of Freestanding Metallic and Semiconducting Nanowires. Small 2014, 10, 725-733.

(8) Chen, B.; Wang, J.; Gao, Q.; Chen, Y. J.; Liao, X. Z.; Lu, C. S.; Tan, H. H.; Mai, Y. W.; Zou, J.; Ringer, S. P.; Gao, H. J.; Jagadish, C. Strengthening Brittle Semiconductor Nanowires through Stacking Faults: Insights from in situ Mechanical Testing. Nano Lett. 2013, 13, 43694373.

(9) Han, X. D.; Zhang, Y. F.; Zheng, K.; Zhang, X. N.; Zhang, Z.; Hao, Y. J.; Guo, X. Y.; Yuan, J.; 
Wang, Z. L. Low-Temperature In Situ Large Strain Plasticity of Ceramic SiC Nanowires and its Atomic-Scale Mechanism. Nano Lett. 2007, 7, 452-457.

(10) Phan, H. P.; Dao, D. V.; Nakamura, K.; Dimitrijev, S.; Nguyen, N. T. The Piezoresistive Effect of $\mathrm{SiC}$ for MEMS Sensors at High Temperatures: A Review. J. Microelectromech. Syst. 2015, $24,1663-1677$.

(11) Melinon, P.; Masenelli, B.; Tournus, F.; Perez, A. Playing with Carbon and Silicon at the Nanoscale. Nat. Mater. 2007, 6, 479-490.

(12) Wu, R. B.; Zhou, K.; Yue, C. Y.; Wei, J.; Pan, Y. Recent Progress in Synthesis, Properties and Potential Applications of SiC Nanomaterials. Prog. Mater. Sci. 2015, 72, 1-60.

(13) Cheng, G. M.; Chang, T. H.; Qin, Q. Q.; Huang, H. C.; Zhu, Y. Mechanical Properties of Silicon Carbide Nanowires: Effect of Size-Dependent Defect Density. Nano Lett. 2014, 14, 754-758.

(14) Chen, J. J.; Liao, X.; Wang, M. M.; Liu, Z. X.; Zhang, J. D.; Ding, L. J.; Gao, L.; Li, Y. Highly Flexible, Nonflammable and Free-Standing SiC Nanowire Paper. Nanoscale 2015, 7, 63746379.

(15) Christle, D. J.; Falk, A. L.; Andrich, P.; Klimov, P. V.; Ul Hassan, J.; Son, N. T.; Janzen, E.; Ohshima, T.; Awschalom, D. D. Isolated Electron Spins in Silicon Carbide with Millisecond Coherence Times. Nat. Mater. 2015, 14, 160-163.

(16) Widmann, M.; Lee, S. Y.; Rendler, T.; Son, N. T.; Fedder, H.; Paik, S.; Yang, L. P.; Zhao, N.; Yang, S.; Booker, I.; Denisenko, A.; Jamali, M.; Momenzadeh, S. A.; Gerhardt, I.; Ohshima, T.; Gali, A.; Janzen, E.; Wrachtrup, J. Coherent Control of Single Spins in Silicon Carbide at Room Temperature. Nat. Mater. 2015, 14, 164-168.

(17) Koehl, W. F.; Buckley, B. B.; Heremans, F. J.; Calusine, G.; Awschalom, D. D. Room Temperature Coherent Control of Defect Spin Qubits in Silicon Carbide. Nature 2011, 479, 8487.

(18) Zhao, S.; Flanagan, R.; Hahn, E. N.; Kad, B.; Remington, B. A.; Wehrenberg, C. E.; Cauble, R.; More, K.; Meyers, M. A. Shock-Induced Amorphization in Silicon Carbide. Acta Mater. 2018, 158, 206-213.

(19) Wang, S. L.; Wu, Y. Q.; Lin, L. W.; He, Y. H.; Huang, H. Fracture Strain of SiC Nanowires and Direct Evidence of Electron-Beam Induced Amorphisation in the Strained Nanowires. Small 
2015, 11, 1672-1676.

(20) Tham, D.; Nam, C. Y.; Fischer, J. E. Microstructure and Composition of Focused-Ion-Beam Deposited Pt Contacts to GaN Nanowires. Adv. Mater. 2006, 18, $290-294$.

(21) Shen, Y. T.; Xu, T.; Tan, X. D.; He, L. B.; Yin, K. B.; Wan, N.; Sun, L. T. In Situ Repair of 2D Chalcogenides under Electron Beam Irradiation. Adv. Mater. 2018, 30, 1705954.

(22) Amjadipour, M.; MacLeod, J.; Lipton-Duffin, J.; Iacopi, F.; Motta, N. Epitaxial Graphene Growth on FIB Patterned 3C-SiC Nanostructures on Si (111): Reducing Milling Damage. Nanotechnology 2017, 28, 345602.

(23) Awaja, F.; Zhang, S. N.; Tripathi, M.; Nikiforov, A.; Pugno, N. Cracks, Microcracks and Fracture in Polymer Structures: Formation, Detection, Autonomic Repair. Prog. Mater. Sci. 2016, $83,536-573$.

(24) Nam, K. W.; Kim, J. S. Critical Crack Size of Healing Possibility of SiC Ceramics. Mater. Sci. Eng. A 2010, 527, 3236-3239.

(25) Osada, T.; Kamoda, K.; Mitome, M.; Hara, T.; Abe, T.; Tamagawa, Y.; Nakao, W.; Ohmura, T. A Novel Design Approach for Self-Crack-Healing Structural Ceramics with 3D Networks of Healing Activator. Sci. Rep. 2017, 7, 17853.

(26) Li, J. X.; Shklyaev, O. E.; Li, T. L.; Liu, W. J.; Shum, H.; Rozen, I.; Balazs, A. C.; Wang, J. Self-Propelled Nanomotors Autonomously Seek and Repair Cracks. Nano Lett. 2015, 15, $7077-7085$.

(27) Guo, C. F.; Lan, Y. C.; Sun, T. Y.; Ren, Z. F. Deformation-Induced Cold-Welding for SelfHealing of Super-Durable Flexible Transparent Electrodes. Nano Energy 2014, 8, 110-117.

(28) Karki, K.; Epstein, E.; Cho, J. H.; Jia, Z.; Li, T.; Picraux, S. T.; Wang, C. S.; Cumings, J. Lithium-Assisted Electrochemical Welding in Silicon Nanowire Battery Electrodes. Nano Lett. 2012, 12, 1392-1397.

(29) Chou, X. J.; Zhu, J.; Qian, S.; Niu, X. S.; Qian, J. C.; Hou, X. J.; Mu, J. L.; Geng, W. P.; Cho, J. D.; He, J.; Xue, C. Y. All-in-one Filler-Elastomer-based High-Performance Stretchable Piezoelectric Nanogenerator for Kinetic Energy Harvesting and Self-Powered Motion Monitoring. Nano Energy 2018, 53, 550-558.

(30) He, J.; Wen, T.; Qian, S.; Zhang, Z. X.; Tian, Z. M.; Zhu, J.; Mu, J. L.; Hou, X. J.; Geng, W. P.; 
Cho, J. D.; Han, J. Q.; Chou, X. J.; Xue, C. Y. Triboelectric-Piezoelectric-Electromagnetic Hybrid Nanogenerator for High-Efficient Vibration Energy Harvesting and Self-Powered Wireless Monitoring System. Nano Energy 2018, 43, 326-339.

(31) Wang, Y. B.; Joyce, H. J.; Gao, Q. A.; Liao, X. Z.; Tan, H. H.; Zou, J.; Ringer, S. P.; Shan, Z. W.; Jagadish, C. Self-Healing of Fractured GaAs Nanowires. Nano Lett. 2011, 11, 1546-1549.

(32) Bechelany, M.; Brioude, A.; Cornu, D.; Ferro, G.; Miele, P. A. Raman Spectroscopy Study of Individual SiC Nanowires. Adv. Funct. Mater. 2007, 17, 939-943.

(33) Yi, J.; Wang, W. H.; Lewandowski, J. J. Sample Size and Preparation Effects on the Tensile Ductility of Pd-Based Metallic Glass Nanowires. Acta Mater. 2015, 87, 1-7.

(34) Kwon, G.; Jo, H. H.; Lim, S.; Shin, C.; Jin, H. H.; Kwon, J.; Sun, G. M. Room-Temperature Yield and Fracture Strength of Single-Crystalline 6H Silicon Carbide. J. Mater. Sci. 2015, 50, 8104-8110.

(35) Nawaz, A.; Mao, W. G.; Lu, C.; Shen, Y. G. Nano-Scale Elastic-Plastic Properties and Indentation-Induced Deformation of Amorphous Silicon Carbide Thin Film. Ceram. Int. 2017, $43,385-391$.

(36) Madar, R. Materials Science - Silicon Carbide in Contention. Nature 2004, 430, 974-975.

(37) Chen, B.; Gao, Q.; Chang, L.; Wang, Y. B.; Chen, Z. B.; Liao, X. Z.; Tan, H. H.; Zou, J.; Ringer, S. P.; Jagadish, C. Attraction of Semiconductor Nanowires: An In Situ Observation. Acta Mater. 2013, 61, 7166-7172.

(38) Lan, Y. W.; Chang, W. H.; Chang, Y. C.; Chang, C. S.; Chen, C. D. Effect of Focused Ion Beam Deposition Induced Contamination on the Transport Properties of Nano Devices. Nanotechnology 2015, 26, 055705.

(39) Ishimaru, M.; Bae, I. T.; Hirotsu, Y. Electron-Beam-Induced Amorphization in SiC. Phys. Rev. B 2003, 68, 144102.

(40) Schulli, T. U.; Daudin, R.; Renaud, G.; Vaysset, A.; Geaymond, O.; Pasturel, A. SubstrateEnhanced Supercooling in AuSi Eutectic Droplets. Nature 2010, 464, 1174-1177.

(41) Pasquarello, A.; Hybertsen, M. S.; Car, R. Interface Structure between Silicon and its Oxide by First-Principles Molecular Dynamics. Nature 1998, 396, 58-60.

(42) Mcmillan, P. F.; Wilson, M.; Daisenberger, D.; Machon, D. A Density-Driven Phase Transition 
between Semiconducting and Metallic Polyamorphs of Silicon. Nat. Mater. 2005, 4, 680-684.

\section{For Table of Contents Only}

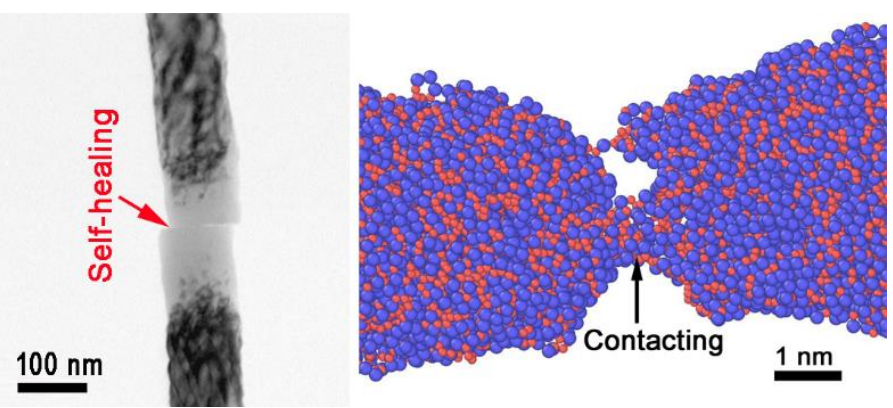

